\title{
LINEAR DIRECT CONNECTIONS
}

\author{
JAN KUBARSKI \\ Institute of Mathematics, Technical University of Łódź \\ Wólczańska 215, 93-005 Lódź, Poland, and \\ Mathematical Institute of Polish Academy of Sciences \\ Śniadeckich 8, 00-950 Warszawa, Poland \\ E-mail:kubarski@p.lodz.pl \\ NICOLAE TELEMAN \\ Dipartimento di Scienze Matematiche, Università Politecnica delle Marche \\ 60161 Ancona, Italy \\ E-mail: teleman@dipmat.univpm.it
}

\begin{abstract}
In this paper we study the geometry of direct connections in smooth vector bundles (see N. Teleman [Tn.3]); we show that the infinitesimal part, $\nabla^{\tau}$, of a direct connection $\tau$ is a linear connection. We determine the curvature tensor of the associated linear connection $\nabla^{\tau}$.

As an application of these results, we present a direct proof of N. Teleman's Theorem 6.2 [Tn.3], which shows that it is possible to represent the Chern character of smooth vector bundles as the periodic cyclic homology class of a specific periodic cyclic cycle $\Phi_{*}^{\tau}$, manufactured from a direct connection $\tau$, rather than from a smooth linear connection as the Chern-Weil construction does. In addition, we show that the image of the cyclic cycle $\Phi_{*}^{\tau}$ into the de Rham cohomology (through the A. Connes' isomorphism) coincides with the cycle provided by the Chern-Weil construction applied to the underlying linear connection $\nabla^{\tau}$.

For more details about these constructions, the reader is referred to $[\mathrm{M}], \mathrm{N}$. Teleman [Th.1], [Tn.2], [Tn.3], C. Teleman [Tc], A. Connes [C.1], [C.2] and A. Connes and H. Moscovici [C.M].
\end{abstract}

1. Introduction. In this paper we address two problems: (i) to better understand the geometry of direct connections and (ii) to provide a direct proof of N. Teleman's theorem [Tn.3], which shows how to modify the Chern-Weil theory from the case of linear connections to the case of direct connections to extract the Chern character of smooth vector bundles.

2000 Mathematics Subject Classification: 53C05, 58A05, 57R20.

This research was supported by the MIUR Contract $N^{\circ} 2005010942 \_002 / 2005$.

The paper is in final form and no version of it will be published elsewhere. 
Before going into more details, we recall that a direct connection (see N. Teleman [Th.2], [Tn.3]) in a vector bundle is a law which provides a direct isomorphic transport of fibres, from point to point, rather than along paths. Direct connections were originally called quasi-connections, see [Tn.2].

Concerning the first problem, we show that any direct connection $\tau$ has an underlying linear connection $\nabla^{\tau}$, which represents its infinitesimal part. We determine the connection coefficients $\Gamma_{\text {.. }}$ of the underlying linear connection and its curvature tensor.

With regard to the second problem, we mention that N. Teleman [Th.3, Th. 6.2] had shown that the Chern character $C h_{*}(\xi)$, of an arbitrary smooth vector bundle $\xi: E \rightarrow M$ may be obtained as the cyclic homology, (resp. periodic cyclic homology), classes, (resp. class) of cyclic cycles, (resp. a periodic cyclic cycle), of the algebra of smooth functions on $M$, whose chain components are the functions

$$
\begin{gathered}
\Phi_{k}: U_{k+1} \rightarrow \mathbb{R}, \\
\Phi_{k}\left(x_{0}, x_{1}, \ldots, x_{k}\right):=\operatorname{Tr} \tau\left(x_{0}, x_{1}\right) \circ \tau\left(x_{1}, x_{2}\right) \circ \cdots \circ \tau\left(x_{k-1}, x_{k}\right) \circ \tau\left(x_{k}, x_{0}\right),
\end{gathered}
$$

where $\tau$ is an arbitrary direct connection in $\xi$ and $U_{k+1}$ is a neighborhood of the diagonal in $M^{k+1}$.

Recall that the periodic cyclic homology of the algebra of smooth functions is isomorphic to the bi-graded de Rham cohomology of the manifold $M$. This result, due to A. Connes, constitutes the bridge between the classical differential geometry and the noncommutative geometry. The Connes' isomorphism associates with any periodic cyclic cycle $f$ an even/odd non-homogeneous closed differential form $\Omega(f)$ on $M$.

The proof of Theorem 6.2. [Th.3] uses the homotopy invariance of the cyclic homology, as well as the non commutative definition of the Chern character derived from the LeviCivita connection in a vector bundle, results due to A. Connes [C.1], [C.2]; it states the result at the level of homology classes.

In this paper we present a direct proof of this theorem, showing in addition that the image of the periodic cyclic cocycle $\left\{\Phi_{k}\right\}_{k}$ through the Connes' isomorphism

$$
\begin{gathered}
\Omega_{2 k}\left(\Phi_{2 k}\right)= \\
\frac{1}{(2 k) !} \sum_{i_{1}, i_{2}, . ., i_{2 k}} \frac{\partial}{\partial x_{1}^{i_{1}}} \frac{\partial}{\partial x_{2}^{i_{2}}} \ldots \frac{\partial}{\partial x_{2 k}^{i_{2 k}}} \Phi_{k}\left(x_{0}, x_{1}, \ldots, x_{2 k}\right)_{x_{0}=x_{1}=\cdots=x_{2 k}=x} d x^{i_{1}} \wedge \cdots \wedge d x^{i_{2 k}}
\end{gathered}
$$

coincides with the differential form provided by the classical Chern-Weil theory applied upon the underlying linear connection $\nabla^{\tau}$.

We recall that within the theory of linear connections the closeness of the form $\operatorname{Tr} R^{k}$ in the de Rham complex is a consequence of the Bianchi identity. It is relevant to mention that the same result follows trivially in the context of direct connections as a consequence of the symmetry of mixed partial derivatives (Schwarz lemma) - see Remark 3.

For other applications of direct connections, we refer to A. Mishchenko and N. Teleman [M.T]. For related topics, providing geometric interpretations of the Chern character, we refer to N. Teleman [Tn.1], [Tn.2], [Tn.3], and C. Teleman [Tc].

In the sequel we freely use the Einstein summation convention on repeated indices. 
2. Direct connections vs. linear connections. Let $E$ be a real or complex smooth vector bundle over the manifold $M$.

Definition 1 (see N. Teleman [Th.2], [Th.3]). By a linear direct connection in a vector bundle $E$ we mean a smooth mapping

$$
\tau: U \rightarrow G L(E)
$$

where $U \subset M \times M$ is an open neighborhood of the diagonal $\Delta=\{(x, x) ; x \in M\}$, such that

$$
\tau(x, y): E_{\mid y} \rightarrow E_{\mid x}
$$

and

$$
\tau(x, x)=\mathrm{id}: E_{\mid x} \rightarrow E_{\mid x} .
$$

Direct connections, used also in the paper by A. Mishchenko and N. Teleman [M.T], were called quasi connections.

We intend to extract from a direct connection its infinitesimal part along the diagonal.

Definition 2. Let $X$ be a smooth tangent field over $M$ and $\phi$ a smooth section in $E$. Let $x_{0}$ be an arbitrary point in $M$ and let $\gamma:(-\varepsilon, \varepsilon) \rightarrow M$ be an integral path of the field $X$ with the initial condition $\gamma(0)=x_{0},\left(\dot{\gamma}(0)=X\left(x_{0}\right)\right)$.

We define

$$
\nabla_{X\left(x_{0}\right)}^{\tau}(\phi)=\frac{d}{d t}\{\tau(\gamma(0), \gamma(t))(\phi(\gamma(t)))\}_{\mid t=0} \in E_{\mid x_{0}} .
$$

REMARK 1. As the parameter $t$ varies, the function under the derivative sign describes a smooth path in the fibre $E_{\mid x_{0}}$ and hence $\nabla_{X\left(x_{0}\right)}^{\tau}(\phi)$ is well defined; it depends only on $X$, $\phi$ and $x_{0}$. Notice that the condition $\tau(x, x)=\mathrm{id}_{E_{x}}$ is necessary in order to insure that the outcome of the derivation is a vector of the fibre over the point $x_{0}$.

We intend to describe $\nabla_{X\left(x_{0}\right)}^{\tau}(\phi)$ locally. For, let $\left(x^{1}, x^{2}, \ldots, x^{m}\right)(\operatorname{dim} M=m)$ be a local coordinate system on an open neighborhood $\mathcal{V}$ of a point $x_{0}$. Using the same local coordinate system on both factors of the direct product $M \times M$, any point $(x, y) \in \mathcal{V} \times \mathcal{V}$ will be given by local coordinates $\left(x^{1}, x^{2}, . ., x^{m} \mid y^{1}, y^{2}, \ldots, y^{m}\right)$.

Let $n$ be the $\mathbb{K}$-rank $\left(\mathbb{K}=\mathbb{R}\right.$, or $\mathbb{C}$ ) of the bundle $E$ and let $\left\{e_{1}, e_{2}, \ldots, e_{n}\right\}$ be a local frame in the bundle $E$ over $V$.

The direct connection $\tau$ is given locally by a matrix

$$
\begin{aligned}
\tau(x \mid y) & =\left\|\tau_{i}^{j}(x \mid y)\right\| \in M_{n, n}(\mathbb{K}), \\
\tau(x \mid y)\left(e_{i}(y)\right) & =\sum_{j} \tau_{i}^{j}(x \mid y) \cdot e_{j}(x), \\
\tau_{i}^{j}(x \mid x) & =\delta_{i}^{j} .
\end{aligned}
$$

The field $\phi$ may be represented over $\mathcal{V}$ by $\phi(x)=\sum_{i} \phi^{i}(x) e_{i}(x)$. Then 


$$
\begin{aligned}
\nabla_{X\left(x_{0}\right)}^{\tau}(\phi) & =\frac{d}{d t}\{\tau(\gamma(0), \gamma(t))(\phi(\gamma(t)))\}_{\mid t=0} \\
& =\frac{d}{d t}\left\{\tau(\gamma(0), \gamma(t))\left(\sum_{i} \phi^{i}(\gamma(t)) e_{i}(\gamma(t))\right)\right\}_{\mid t=0} \\
& =\frac{d}{d t}\left\{\sum_{i, j} \tau_{i}^{j}\left(x_{0} \mid \gamma(t)\right) \cdot \phi^{i}(\gamma(t)) e_{j}\left(x_{0}\right)\right\}_{\mid t=0} \\
& =\sum_{i, j} \frac{d}{d t}\left\{\tau_{i}^{j}\left(x_{0} \mid \gamma(t)\right) \cdot \phi^{i}(\gamma(t))\right\}_{\mid t=0} \cdot e_{j}\left(x_{0}\right) .
\end{aligned}
$$

Describing the path $\gamma$ by $\gamma(t)=\left(y^{1}(t), y^{2}(t), \ldots, y^{m}(t)\right)$, we have further

$$
\begin{aligned}
\nabla_{X\left(x_{0}\right)}^{\tau}(\phi) & =\sum_{i, j} \frac{d}{d t}\left\{\tau_{i}^{j}\left(x_{0} \mid y^{1}(t), y^{2}(t), \ldots, y^{m}(t)\right) \cdot \phi^{i}\left(y^{1}(t), y^{2}(t), \ldots, y^{m}(t)\right)\right\}_{\mid t=0} e_{j}\left(x_{0}\right) \\
= & \sum_{\alpha=1}^{\alpha=m} \sum_{i, j}\left\{\frac{\partial}{\partial y^{\alpha}} \tau_{i}^{j}\left(x_{0} \mid y^{1}, y^{2}, \ldots, y^{m}\right)\right)_{\mid y=x_{0}} \cdot \dot{y}^{\alpha}(0) \cdot \phi^{i}\left(x_{0}\right) \\
& \left.+\tau_{i}^{j}\left(x_{0} \mid x_{0}\right) \frac{\partial}{\partial y^{\alpha}} \phi^{i}\left(y^{1}, y^{2}, \ldots, y^{m}\right)_{\mid y=x_{0}} \cdot \dot{y}^{\alpha}(0)\right\} e_{j}\left(x_{0}\right) .
\end{aligned}
$$

Defining

$$
\left.\Gamma_{i, \alpha}^{j}\left(x_{0}\right)=\frac{\partial}{\partial y^{\alpha}} \tau_{i}^{j}\left(x_{0} \mid y^{1}, y^{2}, \ldots, y^{m}\right)\right)_{\mid y=x_{0}}
$$

we get

$$
\begin{aligned}
& \nabla_{X\left(x_{0}\right)}^{\tau}(\phi) \\
& =\sum_{\alpha=1}^{\alpha=m} \sum_{i, j}\left\{\Gamma_{i, \alpha}^{j}\left(x_{0}\right) \cdot \dot{y}^{\alpha}(0) \cdot \phi^{i}\left(x_{0}\right)+\delta_{i}^{j} \frac{\partial}{\partial y^{\alpha}} \phi^{i}\left(y^{1}, y^{2}, \ldots, y^{m}\right)_{\mid y=x_{0}} \cdot \dot{y}^{\alpha}(0)\right\} e_{j}\left(x_{0}\right) \\
& =\sum_{\alpha=1}^{\alpha=m}\left\{\sum_{i, j} \Gamma_{i, \alpha}^{j}\left(x_{0}\right) \cdot \dot{y}^{\alpha}(0) \cdot \phi^{i}\left(x_{0}\right) e_{j}\left(x_{0}\right)\right. \\
& \left.\quad+\sum_{i} \frac{\partial}{\partial y^{\alpha}} \phi^{i}\left(y^{1}, y^{2}, \ldots, y^{m}\right)_{\mid y=x_{0}} \cdot \dot{y}^{\alpha}(0) e_{i}\left(x_{0}\right)\right\} .
\end{aligned}
$$

Representing the tangent field $X$ locally

$$
X(x)=\sum_{\alpha} X^{\alpha}(x) \cdot \frac{\partial}{\partial x^{\alpha}},
$$

one has $\dot{y}^{\alpha}(0)=X^{\alpha}\left(x_{0}\right)$; the above computation shows that

$$
\begin{aligned}
\nabla_{X\left(x_{0}\right)}^{\tau}\left(\sum_{i} \phi^{i} e_{i}\right) \\
=\sum_{\alpha=1}^{\alpha=m}\left\{\sum_{i, j} \Gamma_{i, \alpha}^{j}\left(x_{0}\right) \cdot X^{\alpha}\left(x_{0}\right) \cdot \phi^{i}\left(x_{0}\right) e_{j}\left(x_{0}\right)\right. \\
\left.\quad+\sum_{i} \frac{\partial}{\partial y^{\alpha}} \phi^{i}\left(y^{1}, y^{2}, \ldots, y^{m}\right)_{\mid y=x_{0}} \cdot X^{\alpha}\left(x_{0}\right) e_{i}\left(x_{0}\right)\right\} \\
=\sum_{\alpha=1}^{\alpha=m}\left\{\sum_{i, j} \Gamma_{i, \alpha}^{j}\left(x_{0}\right) \cdot X^{\alpha}\left(x_{0}\right) \cdot \phi^{i}\left(x_{0}\right) e_{j}\left(x_{0}\right)\right\}+\sum_{i}\left(d \phi^{i}\right)(X)\left(x_{0}\right) e_{i}\left(x_{0}\right) .
\end{aligned}
$$


Both this formula and Remark 1 above show that $\nabla^{\tau}$ is a linear connection in the vector bundle $E$. The linear connection $\nabla^{\tau}$ will be called associated, or underlying, linear connection to the direct connection $\tau$. To summarize, we have proved

THEOREM 3. (i) For any direct connection $\tau$, let

$$
\nabla_{X(x)}^{\tau}(\phi)=\frac{d}{d t} \tau(\gamma(0), \gamma(t))_{\mid t=0}(\phi(\gamma(t))) \in E_{\mid x}, \quad \gamma(0)=x, \quad \dot{\gamma}(t)=X(\gamma(t))
$$

be its infinitesimal part along the diagonal. Then $\nabla^{\tau}$ is a linear connection.

(ii) Let $\left(x^{1}, x^{2}, \ldots, x^{m} \mid y^{1}, y^{2}, \ldots, y^{m}\right)$ be a local system of coordinates on a neighborhood $\mathcal{V} \times \mathcal{V}$ of a point $(x, x) \in M \times M$ and let $\left\{e_{1}, e_{2}, \ldots, e_{n}\right\}$ be a local frame in $E$ over $\mathcal{V}$. Let $\tau(x \mid y)$ be the matrix describing locally the direct connection $\tau$ :

$$
\begin{gathered}
\tau(x \mid y)=\left\|\tau_{i}^{j}(x \mid y)\right\| \in M_{n, n}(\mathbb{K}), \\
\tau(x, y)\left(e_{i}(y)\right)=\sum_{j} \tau_{i}^{j}(x \mid y) \cdot e_{j}(x), \quad \tau_{i}^{j}(x \mid x)=\delta_{i}^{j} .
\end{gathered}
$$

Then the coefficients $\Gamma_{i, \alpha}^{j}$ of the connection $\nabla^{\tau}$ are given locally by

$$
\nabla_{\frac{\partial}{\partial x^{\alpha}}}^{\tau} e_{i}=\sum_{j} \Gamma_{i, \alpha}^{j} e_{j}
$$

where

$$
\Gamma_{i, \alpha}^{j}(x)=\frac{\partial}{\partial y^{\alpha}} \tau_{i}^{j}\left(x^{1}, x^{2}, \ldots, x^{m} \mid y^{1}, y^{2}, \ldots, y^{m}\right)_{y=x} .
$$

In the above formulas it is assumed that on each of the two factors $\mathcal{V}$ of $\mathcal{V} \times \mathcal{V}$ the same coordinate functions are considered.

Let $R=\left(\nabla^{\tau}\right)^{2}$ be the curvature tensor of the connection $\nabla^{\tau}$. The components of the curvature $R$ are

$$
\begin{aligned}
R_{i \alpha \beta}^{j}(x)= & \frac{\partial}{\partial x^{\alpha}} \Gamma_{i \beta}^{j}(x)-\frac{\partial}{\partial x^{\beta}} \Gamma_{i \alpha}^{j}(x)+\Gamma_{k \alpha}^{j}(x) \cdot \Gamma_{i \beta}^{k}(x)-\Gamma_{k \beta}^{j}(x) \cdot \Gamma_{i \alpha}^{k}(x) \\
= & \frac{\partial^{2}}{\partial x^{\alpha} \partial y^{\beta}} \tau_{i}^{j}(x \mid y)_{y=x}-\frac{\partial^{2}}{\partial x^{\beta} \partial y^{\alpha}} \tau_{i}^{j}(x \mid y)_{y=x} \\
& +\frac{\partial}{\partial y^{\alpha}} \tau_{k}^{j}(x \mid y)_{y=x} \cdot \frac{\partial}{\partial y^{\beta}} \tau_{i}^{k}(x \mid y)_{y=x}-\frac{\partial}{\partial y^{\beta}} \tau_{k}^{j}(x \mid y)_{y=x} \cdot \frac{\partial}{\partial y^{\alpha}} \tau_{i}^{k}(x \mid y)_{y=x} .
\end{aligned}
$$

COROLLARY 4. The curvature form $R$ of the underlying linear connection $\nabla^{\tau}$, associated to the direct connection $\tau$, is given by

$$
\begin{aligned}
R= & \left(\frac{\partial^{2}}{\partial x^{\alpha} \partial y^{\beta}} \tau_{i}^{j}(x \mid y)_{y=x}-\frac{\partial^{2}}{\partial x^{\beta} \partial y^{\alpha}} \tau_{i}^{j}(x \mid y)_{y=x}\right. \\
& \left.+\frac{\partial}{\partial y^{\alpha}} \tau_{k}^{j}(x \mid y)_{y=x} \cdot \frac{\partial}{\partial y^{\beta}} \tau_{i}^{k}(x \mid y)_{y=x}-\frac{\partial}{\partial y^{\beta}} \tau_{k}^{j}(x \mid y)_{y=x} \cdot \frac{\partial}{\partial y^{\alpha}} \tau_{i}^{k}(x \mid y)_{y=x}\right) d x^{\alpha} \wedge d x^{\beta} .
\end{aligned}
$$

For further computations it is convenient to introduce the matrices $\Gamma_{\alpha}$ whose components are given by

$$
\left\|\Gamma_{\alpha}\right\|_{i}^{j}:=\Gamma_{i, \alpha}^{j}
$$

and matrices $R_{\alpha \beta}$ whose entries are the components of the curvature tensor

$$
\left\|R_{\alpha \beta}\right\|_{i}^{j}:=R_{i \alpha \beta}^{j} .
$$


REMARK 2. It is clear that if the base manifold $M$ is endowed with an affine connection, then the parallel transport along small geodesics-defined for a given linear connection in the vector bundle $E$ over $M$-produces a direct connection $\tau$ in $E$. Such examples of direct connections have already been used in various papers, as computational tools, see e.g. C. Teleman [Tc], A. Connes and H. Moscovici [C.M]. We might ask whether any direct connection can be obtained by this procedure. The answer to this question is negative. Indeed, notice that any direct connection $\tau$ produced by parallel transport along geodesics has to satisfy the condition $\tau(x, y) \circ \tau(y, x)=I d$, a property which might not be satisfied by an arbitrary direct connection. Moreover, if a direct connection $\tau$ derives from the parallel transport along geodesics, then the affine connection on the base manifold might not be uniquely defined by it. For example, if the linear connection in $E$ is flat, then the corresponding direct connection $\tau$ satisfies the condition $\tau(z, y) \circ \tau(y, x)=\tau(z, x)$, locally, and it does not depend on the affine connection on the base manifold.

It is interesting to point out that the fulfillment of the condition $\tau(x, y) \circ \tau(y, x)=I d$ is not sufficient to insure that the direct connection $\tau$ derives from the parallel transport along geodesics, either. For, we provide the following

EXAmple 5 . Let $M=\mathbb{R}$ and let $E$ be the product bundle $M \times \mathbb{R}$ of rank 1 . Let $\mathbf{e}_{1}$ be the constant frame $\mathbf{e}_{1}(x)=(x, 1)$ in $E$. With respect to this frame, consider the direct connection $\tau$ defined by the matrix

$$
\tau(y \mid x)=e^{y-x+(y-x)^{3}} \in M_{1,1}(\mathbb{R}) .
$$

As the exponent is an odd function, $\tau$ satisfies $\tau(x, y) \circ \tau(y, x)=I d$. On the other hand, the corresponding linear connection $\nabla^{\tau}$ is given by

$$
\nabla_{\frac{d}{d x}}^{\tau}\left(\mathbf{e}_{1}(x)\right)=\frac{d}{d y}\left(e^{y-x+(y-x)^{3}} \mathbf{e}_{1}(x)\right)_{y=x}=\mathbf{e}_{1}(x)
$$

and the parallel transport of the vector $\xi_{0}$ along the line $\mathbb{R}$ from the point $x$ to the point $y$ is the solution of the differential equation

$$
\frac{d \xi(y)}{d y}=\xi(y), \quad \xi(x)=\xi_{0}
$$

or,

$$
\xi(y)=e^{y-x} \xi_{0},
$$

which differs from $e^{y-x+(y-x)^{3}} \xi_{0}$.

Although $\tau(x, y)=(\tau(y, x))^{-1}$ is not true in general, it is true, however, that it holds infinitesimally. In fact, we have

Proposition 6. For any direct connection $\tau$, its matrix components satisfy the identities (i)

$$
\frac{\partial}{\partial x^{\alpha}} \tau_{i}^{j}(x \mid y)_{y=x}+\frac{\partial}{\partial y^{\alpha}} \tau_{i}^{j}(x \mid y)_{y=x}=0
$$

$$
\frac{\partial}{\partial x^{\alpha}}\{\tau(x \mid y) \circ \tau(y \mid x)\}_{y=x}=0=\frac{\partial}{\partial y^{\alpha}}\{\tau(x \mid y) \circ \tau(y \mid x)\}_{y=x}
$$


Proof. As $\tau(x \mid x)=I d$, we get that the directional derivative $\left(\frac{\partial}{\partial x^{\alpha}}+\frac{\partial}{\partial y^{\alpha}}\right)$ of $\tau$ along the diagonal vanishes. This proves (i). The second identity is a consequence of the first.

\section{Direct connections and Chern character}

3.1. Recall of periodic cyclic homology. For the benefit of the reader and for setting notation, we recall in this section some basic notions and results, due to A. Connes [C.1], [C.2], which lay at the foundations of noncommutative geometry.

Given a locally convex associative algebra $\mathcal{A}$, the space of $k$-chains $C_{k}(\mathcal{A})$ over the algebra $\mathcal{A}, C_{*}(\mathcal{A})$ is, by definition, a topological completion (usually, projective completion) of the algebraic tensor product $\otimes^{k+1} \mathcal{A}$. Two boundary operators, $b^{\prime}$ and $b$ are introduced by the formulas

$$
b^{\prime}\left(f_{0} \otimes f_{1} \otimes \ldots f_{k-1} \otimes f_{k}\right)=\sum_{r=0}^{r=k-1}(-1)^{r} f_{0} \otimes f_{1} \otimes \cdots \otimes\left(f_{r} \cdot f_{r+1}\right) \otimes \cdots \otimes f_{k-1} \otimes f_{k}
$$

and

$$
\begin{aligned}
b\left(f_{0} \otimes f_{1} \otimes \ldots f_{k-1} \otimes f_{k}\right)= & \sum_{r=0}^{r=k-1}(-1)^{r} f_{0} \otimes f_{1} \otimes \cdots \otimes\left(f_{r} \cdot f_{r+1}\right) \otimes \cdots \otimes f_{k-1} \otimes f_{k} \\
& +(-1)^{k} f_{0} \cdot f_{1} \otimes \cdots \otimes f_{k-1} .
\end{aligned}
$$

The boundary operator $b^{\prime}$ defines the bar complex; if the algebra $\mathcal{A}$ is unitary then the bar complex is acyclic.

The complex based on the boundary operator $b$ is the Hochschild complex of the algebra $\mathcal{A}$; its homology is the Hochschild homology of the algebra.

The graded cyclic permutation $T: C_{k}(\mathcal{A}) \rightarrow C_{k}(\mathcal{A})$ is defined on generators by

$$
T\left(f_{0} \otimes f_{1} \otimes \cdots \otimes f_{k-1} \otimes f_{k}\right)=(-1)^{k} f_{1} \otimes \cdots \otimes f_{k-1} \otimes f_{k} \otimes f_{0} .
$$

The operator $N: C_{k}(\mathcal{A}) \rightarrow C_{k}(\mathcal{A})$ is given by

$$
N=1+T+T^{2}+\cdots+T^{k} .
$$

The periodic cyclic homology is defined as the homology of the total complex associated to a first and second quadrant direct product bicomplex $\left\{C_{p, q}\right\}_{p \in Z, q \geq 0}$ defined by:

(i) $C_{p, q}=C_{q}(\mathcal{A})$; the boundary operators are considered of degree -1 ,

(ii) the columns consist of alternating bar and Hochschild complexes: the Hochschild complex on each even order column and the bar complex (with $b^{\prime}$ replaced by $-b^{\prime}$ ) on each odd order column,

(iii) the boundary homomorphisms of the horizontal complexes are given by the alternating homomorphisms $N$ and $1-T$

$$
\ldots \stackrel{1-T}{\longleftarrow} C_{-1, q} \stackrel{N}{\longleftarrow} C_{0, q} \stackrel{1-T}{\longleftarrow} C_{1, q} \stackrel{N}{\longleftarrow} \ldots
$$

This complex is called the periodic bicomplex of the algebra $\mathcal{A}$.

Given the periodicity of the periodic cyclic bicomplex, there are essentially only two periodic cyclic homologies: $H_{\text {even }}^{\lambda, p e r}(\mathcal{A})$, and $H_{\text {odd }}^{\lambda, p e r}(\mathcal{A})$. 
Theorem 7 (A. Connes [C.1], [C.2]).

(i)

$$
H_{\text {even }}^{\lambda, p e r}\left(C^{\infty}(M)\right)=\bigoplus^{k=e v e n} H_{d R}^{k}(M),
$$

(ii)

$$
H_{\text {odd }}^{\lambda, p e r}\left(C^{\infty}(M)\right)=\bigoplus^{k=o d d} H_{d R}^{k}(M)
$$

(iii) Given the periodic cyclic cycle $f=\prod_{p=0}^{p=\infty} f_{\epsilon-p, p} \in \prod_{p=0}^{p=\infty} C_{\epsilon-p, p}\left(C^{\infty}(M)\right)$ $(\epsilon=0,1)$, its $(\epsilon+2 p)$-degree component is the de Rham cohomology class of the (closed) differential form

$$
\frac{1}{(\epsilon+2 p) !} \frac{\partial^{\epsilon+2 p}}{\partial x_{1}^{i_{1}} \ldots \partial x_{\epsilon+2 p}^{i_{\epsilon+2 p}}} f_{\epsilon+2 p}\left(x_{0}, x_{1}, \ldots, x_{\epsilon+2 p}\right)_{\mid \Delta} d x_{1}^{i_{1}} \wedge \cdots \wedge d x_{\epsilon+2 p}^{i_{\epsilon+2 p}},
$$

where $\Delta$ is the diagonal.

Definition 8. For any cyclic cycle $f=\prod_{p=0}^{p=\infty} f_{\epsilon-p, p}$, let

$$
\Omega_{\epsilon+2 p}(f):=\frac{1}{(\epsilon+2 p) !} \frac{\partial^{\epsilon+2 p}}{\partial x_{1}^{i_{1}} \ldots \partial x_{\epsilon+2 p}^{i_{\epsilon+2 p}}} f_{\epsilon+2 p}\left(x_{0}, x_{1}, \ldots, x_{\epsilon+2 p}\right)_{\mid \Delta} d x_{1}^{i_{1}} \wedge \cdots \wedge d x_{\epsilon+2 p}^{i_{\epsilon+2 p}} .
$$

3.2. Direct connections and Chern character forms. Let $\tau$ be a direct connection in the complex vector bundle $\xi: E \rightarrow M$ and let $\nabla^{\tau}$ be its associated linear connection.

Consider the function $\Phi_{k}: M^{k+1} \supset U_{k+1} \rightarrow \mathbb{C}$ (where $U_{k+1} \subset M^{k+1}$ is a neighborhood of the diagonal in $M^{k+1}$ ) defined by the formula

$$
\Phi_{k}\left(x_{0}, x_{1}, \ldots, x_{k}\right):=\operatorname{Tr} \tau\left(x_{0}, x_{1}\right) \tau\left(x_{1}, x_{2}\right) \ldots \tau\left(x_{k-1}, x_{k}\right) \tau\left(x_{k}, x_{0}\right) .
$$

Theorem 9 (N. Teleman, [Tn.2] Theorem 6.2). Let $\xi$ be a complex vector bundle over the paracompact manifold $M$ and let $\tau$ be a smooth linear direct connection in $\xi$. Then

(i) the infinite chain $\Phi^{\tau}$ with components

$$
\begin{gathered}
f_{-2 p, 2 p}:=(-1)^{p} \frac{(2 p) !}{p !} \Phi_{2 p} \in C_{-2 p, 2 p}\left(C^{\infty}(M)\right) \\
f_{-(2 p-1), 2 p-1}:=(-1)^{p-1} \frac{(2 p) !}{p !} \Phi_{2 p-1} \in C_{-(2 p-1), 2 p-1}\left(C^{\infty}(M)\right)
\end{gathered}
$$

is an even periodic cyclic cycle over the algebra $C^{\infty}(M)$;

(ii) its homology class is (up to a multiplicative constants) the total Chern character of $\xi$.

The reader should notice that, in view of Connes' result, Theorem 7, a modification of the direct connection away from a small neighborhood of the diagonal does not change the periodic cyclic homology class of the chain $\Phi^{\tau}$ as its periodic cyclic homology class depends only on its 1-jet along the diagonal. For more information about the Hochschild homology of the algebra of smooth functions, the reader might refer to N. Teleman [Tn.4]

As explained in the section 1, we intend to present here a direct proof of this theorem providing, in addition, an explicite link between the differential forms $\Omega_{2 k}\left(\Phi^{\tau}\right)$ and the classical Chern-Weil forms, at the level of differential forms rather than cohomology classes. 
More precisely, we prove

THEOREM 10. Let $\tau$ be a direct connection and let $\nabla^{\tau}$ be its underlying linear connection. Then

$$
\Omega_{2 k}\left(\Phi_{2 k}^{\tau}\right)=\frac{1}{(2 k) !} \cdot \frac{1}{2^{k}} \cdot \operatorname{Tr} R^{k}
$$

where $R=\left(\nabla^{\tau}\right)^{2}$ is the curvature of the underlying linear connection $\nabla^{\tau}$.

Proof. We have to evaluate

$$
\Omega_{2 k}\left(\Phi_{2 k}^{\tau}\right):=
$$

$$
\sum_{i_{1}, \ldots, i_{2 k}} \frac{1}{(2 k) !} \frac{\partial^{2 k}}{\partial x_{1}^{i_{1}} \ldots \partial x_{2 k}^{i_{2 k}}} \operatorname{Tr} \tau\left(x_{0}, x_{1}\right) \ldots \tau\left(x_{2 k-1}, x_{2 k}\right) \tau\left(x_{2 k}, x_{0}\right)_{\mid \Delta} d x_{0}^{i_{1}} \wedge \cdots \wedge d x_{0}^{i_{2 k}} .
$$

We intend to evaluate the differential form $\Omega_{2 k}\left(\Phi_{2 k}^{\tau}\right)$ at the point $\left(x_{0}, x_{0}, \ldots, x_{0}\right)$.

LEMMA 11.

$$
\begin{aligned}
& \sum_{i_{1}, i_{2}} \frac{\partial^{2}}{\partial x_{1}^{i_{1}} \partial x_{2}^{i_{2}}}\left(\tau\left(x_{0}, x_{1}\right) \tau\left(x_{1}, x_{2}\right) \tau\left(x_{2}, x_{3}\right)\right)_{\mid\left(x_{0}=x_{1}=x_{2}\right)} d x_{0}^{i_{1}} \wedge d x_{0}^{i_{2}} \\
& =\frac{1}{2} \sum_{i_{1}, i_{2}} R_{i_{1} i_{2}}\left(x_{0}\right) \tau\left(x_{0} \mid x_{3}\right) d x_{0}^{i_{1}} \wedge d x_{0}^{i_{2}} .
\end{aligned}
$$

Proof. We work in local coordinates. Let us define

$$
A\left(x_{0}, x_{3}\right):=\sum_{i_{1}, i_{2}} \frac{\partial^{2}}{\partial x_{1}^{i_{1}} \partial x_{2}^{i_{2}}}\left(\tau\left(x_{0}, x_{1}\right) \tau\left(x_{1}, x_{2}\right) \tau\left(x_{2}, x_{3}\right)\right)_{\mid\left(x_{0}=x_{1}=x_{2}\right)} d x_{0}^{i_{1}} \wedge d x_{0}^{i_{2}} .
$$

An elementary computation gives

$$
\begin{aligned}
& A\left(x_{0}, x_{3}\right) \\
& =\left\{\left(\frac{\partial}{\partial x_{1}^{i_{1}}} \tau\left(x_{0} \mid x_{1}\right)\right) \cdot\left(\frac{\partial}{\partial x_{2}^{i_{2}}} \tau\left(x_{1} \mid x_{2}\right)\right) \cdot \tau\left(x_{2} \mid x_{3}\right)\right. \\
& +\left(\frac{\partial}{\partial x_{1}^{i_{1}}} \tau\left(x_{0} \mid x_{1}\right)\right) \cdot \tau\left(x_{1} \mid x_{2}\right) \cdot\left(\frac{\partial}{\partial x_{2}^{i_{2}}} \tau\left(x_{2} \mid x_{3}\right)\right) \\
& +\tau\left(x_{0} \mid x_{1}\right) \cdot\left(\frac{\partial}{\partial x_{1}^{i_{1}}} \frac{\partial}{\partial x_{2}^{i_{2}}} \tau\left(x_{1} \mid x_{2}\right)\right) \cdot \tau\left(x_{2} \mid x_{3}\right) \\
& \left.+\tau\left(x_{0} \mid x_{1}\right) \cdot\left(\frac{\partial}{\partial x_{1}^{i_{1}}} \tau\left(x_{1} \mid x_{2}\right)\right) \cdot\left(\frac{\partial}{\partial x_{2}^{i_{2}}} \tau\left(x_{2} \mid x_{3}\right)\right)\right\}_{\mid\left(x_{0}=x_{1}=x_{2}\right)} d x_{0}^{i_{1}} \wedge d x_{0}^{i_{2}} \\
& =\left\{\left(\frac{\partial}{\partial x_{1}^{i_{1}}} \tau\left(x_{0} \mid x_{1}\right)\right) \cdot\left(\frac{\partial}{\partial x_{2}^{i_{2}}} \tau\left(x_{1} \mid x_{2}\right)\right) \cdot \tau\left(x_{0} \mid x_{3}\right)+\left(\frac{\partial}{\partial x_{1}^{i_{1}}} \tau\left(x_{0} \mid x_{1}\right)\right) \cdot\left(\frac{\partial}{\partial x_{2}^{i_{2}}} \tau\left(x_{2} \mid x_{3}\right)\right)\right. \\
& +\left(\frac{\partial}{\partial x_{1}^{i_{1}}} \frac{\partial}{\partial x_{2}^{i_{2}}} \tau\left(x_{1} \mid x_{2}\right)\right) \cdot \tau\left(x_{0} \mid x_{3}\right) \\
& \left.+\left(\frac{\partial}{\partial x_{1}^{i_{1}}} \tau\left(x_{1} \mid x_{0}\right)\right) \cdot\left(\frac{\partial}{\partial x_{2}^{i_{2}}} \tau\left(x_{2} \mid x_{3}\right)\right)\right\}_{\mid\left(x_{0}=x_{1}=x_{2}\right)} d x_{0}^{i_{1}} \wedge d x_{0}^{i_{2}} .
\end{aligned}
$$


Using Proposition 6 and the notation for the matrices $\Gamma_{\alpha}, R_{\alpha \beta}$, we have further

$$
\begin{aligned}
A\left(x_{0}, x_{3}\right)= & \left\{\Gamma_{i_{1}}\left(x_{0}\right) \cdot \Gamma_{i_{2}}\left(x_{0}\right) \cdot \tau\left(x_{0} \mid x_{3}\right)+\left(\frac{\partial}{\partial x_{1}^{i_{1}}} \tau\left(x_{0} \mid x_{1}\right)\right) \cdot\left(\frac{\partial}{\partial x_{2}^{i_{2}}} \tau\left(x_{2} \mid x_{3}\right)\right)\right. \\
& +\left(\frac{\partial}{\partial x_{1}^{i_{1}}} \frac{\partial}{\partial x_{2}^{i_{2}}} \tau\left(x_{1} \mid x_{2}\right)\right) \cdot \tau\left(x_{0} \mid x_{3}\right) \\
& \left.+\left(\frac{\partial}{\partial x_{1}^{i_{1}}} \tau\left(x_{1} \mid x_{0}\right)\right) \cdot\left(\frac{\partial}{\partial x_{2}^{i_{2}}} \tau\left(x_{2} \mid x_{3}\right)\right)\right\}_{\mid\left(x_{0}=x_{1}=x_{2}\right)} d x_{0}^{i_{1}} \wedge d x_{0}^{i_{2}} \\
= & \left\{\left(\Gamma_{i_{1}}\left(x_{0}\right) \cdot \Gamma_{i_{2}}\left(x_{0}\right)+\frac{\partial}{\partial x_{1}^{i_{1}}} \frac{\partial}{\partial x_{2}^{i_{2}}} \tau\left(x_{1} \mid x_{2}\right)\right) \cdot \tau\left(x_{0} \mid x_{3}\right)\right. \\
& \left.\left.+\left(\frac{\partial}{\partial x_{1}^{i_{1}}} \tau\left(x_{0} \mid x_{1}\right)+\frac{\partial}{\partial x_{1}^{i_{1}}} \tau\left(x_{1} \mid x_{0}\right)\right) \cdot \frac{\partial}{\partial x_{2}^{i_{2}}} \tau\left(x_{2} \mid x_{3}\right)\right)\right\}_{\mid\left(x_{0}=x_{1}=x_{2}\right)} d x_{0}^{i_{1}} \wedge d x_{0}^{i_{2}} .
\end{aligned}
$$

From Corollary 4 and Proposition 6, we get

$$
\begin{aligned}
A\left(x_{0}, x_{3}\right) & \sum_{i_{1}, i_{2}}\left\{\left(\Gamma_{i_{1}}\left(x_{0}\right) \cdot \Gamma_{i_{2}}\left(x_{0}\right)+\frac{\partial}{\partial x_{1}^{i_{1}}} \frac{\partial}{\partial x_{2}^{i_{2}}} \tau\left(x_{1} \mid x_{2}\right)\right) \cdot \tau\left(x_{0} \mid x_{3}\right)\right\}_{\mid\left(x_{0}=x_{1}=x_{2}\right)} d x_{0}^{i_{1}} \wedge d x_{0}^{i_{2}} \\
= & \frac{1}{2} \sum_{i_{1}, i_{2}}\left\{\left(\Gamma_{i_{1}}\left(x_{0}\right) \cdot \Gamma_{i_{2}}\left(x_{0}\right)+\frac{\partial}{\partial x_{1}^{i_{1}}} \frac{\partial}{\partial x_{2}^{i_{2}}} \tau\left(x_{1} \mid x_{2}\right)\right) \cdot \tau\left(x_{0} \mid x_{3}\right)\right\}_{\mid\left(x_{0}=x_{1}=x_{2}\right)} d x_{0}^{i_{1}} \wedge d x_{0}^{i_{2}} \\
& -\frac{1}{2} \sum_{i_{1}, i_{2}}\left\{\left(\Gamma_{i_{1}}\left(x_{0}\right) \cdot \Gamma_{i_{2}}\left(x_{0}\right)+\frac{\partial}{\partial x_{1}^{i_{1}}} \frac{\partial}{\partial x_{2}^{i_{2}}} \tau\left(x_{1} \mid x_{2}\right)\right) \cdot \tau\left(x_{0} \mid x_{3}\right)\right\}_{\mid\left(x_{0}=x_{1}=x_{2}\right)} d x_{0}^{i_{2}} \wedge d x_{0}^{i_{1}} \\
= & \frac{1}{2} \sum_{i_{1}, i_{2}}\left\{\left(\Gamma_{i_{1}}\left(x_{0}\right) \cdot \Gamma_{i_{2}}\left(x_{0}\right)+\frac{\partial}{\partial x_{1}^{i_{1}}} \frac{\partial}{\partial x_{2}^{i_{2}}} \tau\left(x_{1} \mid x_{2}\right)\right) \cdot \tau\left(x_{0} \mid x_{3}\right)\right\}_{\mid\left(x_{0}=x_{1}=x_{2}\right)} d x_{0}^{i_{1}} \wedge d x_{0}^{i_{2}} \\
& -\frac{1}{2} \sum_{i_{1}, i_{2}}\left\{\left(\Gamma_{i_{2}}\left(x_{0}\right) \cdot \Gamma_{i_{1}}\left(x_{0}\right)+\frac{\partial}{\partial x_{1}^{i_{2}}} \frac{\partial}{\partial x_{2}^{i_{1}}} \tau\left(x_{1} \mid x_{2}\right)\right) \cdot \tau\left(x_{0} \mid x_{3}\right)\right\}_{\mid\left(x_{0}=x_{1}=x_{2}\right)} d x_{0}^{i_{1}} \wedge d x_{0}^{i_{2}} \\
= & \frac{1}{2} \sum_{i_{1}, i_{2}} R_{i_{1} i_{2}} \cdot \tau\left(x_{0} \mid x_{3}\right)_{\mid\left(x_{0}=x_{1}=x_{2}\right)} d x_{0}^{i_{1}} \wedge d x_{0}^{i_{2}} \cdot
\end{aligned}
$$

This completes the proof of the Lemma.

LEMMA 12.

$$
\begin{gathered}
\operatorname{Tr} \frac{\partial^{2 k}}{\partial x_{1}^{i_{1}} \ldots \partial x_{2 k}^{i_{2 k}}} \tau\left(x_{0}, x_{1}\right) \ldots \tau\left(x_{2 k-1}, x_{2 k}\right) \tau\left(x_{2 k}, x_{0}\right)_{\mid \Delta} \cdot\left(d x_{0}^{i_{1}} \wedge d x_{0}^{i_{2}}\right) \otimes \cdots \otimes\left(d x_{0}^{i_{2 k-1}} \wedge d x_{0}^{i_{2 k}}\right) \\
=\frac{1}{2^{k}} \sum_{i_{1}, i_{2}, \ldots, i_{2 k-1}, i_{2 k}} \operatorname{Tr} R_{i_{1} i_{2}}\left(x_{0}\right) R_{i_{3} i_{4}}\left(x_{0}\right) \ldots R_{i_{2 k-1} i_{2 k}}\left(x_{0}\right) \\
\cdot\left(d x_{0}^{i_{1}} \wedge d x_{0}^{i_{2}}\right) \otimes \cdots \otimes\left(d x_{0}^{i_{2 k-1}} \wedge d x_{0}^{i_{2 k}}\right) .
\end{gathered}
$$

Proof. We apply Lemma 11, in succession, to each of the pairs of arguments and corresponding partial derivations, beginning with the first pair $\left(x_{1}, x_{2}\right)$. This procedure, followed by applying the trace operator, leads to the desired relation. 
We return to the proof of the theorem. Denoting by Alt the skew-symmetrization of tensor products

$$
\operatorname{Alt}\left(v_{1} \otimes v_{2} \otimes \cdots \otimes v_{r}\right):=\frac{1}{r !} \sum_{\iota \in S_{r}} \operatorname{Sign}(\iota) \cdot v_{\iota_{1}} \otimes v_{\iota_{2}} \otimes \cdots \otimes v_{\iota_{r}}:=v_{1} \wedge v_{2} \wedge \cdots \wedge v_{r},
$$

we have

$$
\begin{aligned}
& (2 k) ! \cdot \Omega_{2 k}\left(\Phi_{2 k}^{\tau}\right) \\
& :=\frac{\partial^{2 k}}{\partial x_{1}^{i_{1}} \ldots \partial x_{2 k}^{i_{2 k}}} \operatorname{Tr} \tau\left(x_{0}, x_{1}\right) \ldots \tau\left(x_{2 k-1}, x_{2 k}\right) \tau\left(x_{2 k}, x_{0}\right)_{\mid \Delta} d x_{0}^{i_{1}} \wedge \cdots \wedge d x_{0}^{i_{2 k}} \\
& =\frac{\partial^{2 k}}{\partial x_{1}^{i_{1}} \ldots \partial x_{2 k}^{i_{2 k}}} \operatorname{Tr} \tau\left(x_{0}, x_{1}\right) \ldots \tau\left(x_{2 k-1}, x_{2 k}\right) \tau\left(x_{2 k}, x_{0}\right)_{\mid \Delta} A l t\left(d x_{0}^{i_{1}} \otimes \cdots \otimes d x_{0}^{i_{2 k}}\right) \\
& \left.=\frac{\partial^{2 k}}{\partial x_{1}^{i_{1}} \ldots \partial x_{2 k}^{i_{2 k}}} \operatorname{Tr} \tau\left(x_{0}, x_{1}\right) \ldots \tau\left(x_{2 k}, x_{0}\right)_{\mid \Delta} A l t\left[\left(d x_{0}^{i_{1}} \wedge d x_{0}^{i_{2}}\right) \otimes \cdots \otimes\left(d x_{0}^{i_{2 k-1}} \wedge d x_{0}^{i_{2 k}}\right)\right)\right] \\
& \left.=A l t\left[\frac{\partial^{2 k}}{\partial x_{1}^{i_{1}} \ldots \partial x_{2 k}^{i_{2 k}}} \operatorname{Tr} \tau\left(x_{0}, x_{1}\right) \ldots \tau\left(x_{2 k}, x_{0}\right)_{\mid \Delta}\left(d x_{0}^{i_{1}} \wedge d x_{0}^{i_{2}}\right) \otimes \cdots \otimes\left(d x_{0}^{i_{2 k-1}} \wedge d x_{0}^{i_{2 k}}\right)\right)\right] \\
& =\frac{1}{2^{k}} \cdot \operatorname{Alt}\left[\operatorname{Tr} R_{i_{1} i_{2}}\left(x_{0}\right) R_{i_{3} i_{4}}\left(x_{0}\right) \ldots R_{i_{2 k-1} i_{2 k}}\left(x_{0}\right) \cdot\left(d x_{0}^{i_{1}} \wedge d x_{0}^{i_{2}}\right) \otimes \cdots \otimes\left(d x_{0}^{i_{2 k-1}} \wedge d x_{0}^{i_{2 k}}\right)\right] \\
& =\frac{1}{2^{k}} \cdot \operatorname{Tr} R_{i_{1} i_{2}}\left(x_{0}\right) R_{i_{3} i_{4}}\left(x_{0}\right) \ldots R_{i_{2 k-1} i_{2 k}}\left(x_{0}\right) \cdot \operatorname{Alt}\left[\left(d x_{0}^{i_{1}} \wedge d x_{0}^{i_{2}}\right) \otimes \cdots \otimes\left(d x_{0}^{i_{2 k-1}} \wedge d x_{0}^{i_{2 k}}\right)\right] \\
& =\frac{1}{2^{k}} \cdot \operatorname{Tr} R_{i_{1} i_{2}}\left(x_{0}\right) R_{i_{3} i_{4}}\left(x_{0}\right) \ldots R_{i_{2 k-1} i_{2 k}}\left(x_{0}\right) \cdot \operatorname{Alt}\left[\left(d x_{0}^{i_{1}} \otimes d x_{0}^{i_{2}}\right) \otimes \cdots \otimes\left(d x_{0}^{i_{2 k-1}} \otimes d x_{0}^{i_{2 k}}\right)\right] \\
& =\frac{1}{2^{k}} \cdot \operatorname{Tr} R_{i_{1} i_{2}}\left(x_{0}\right) R_{i_{3} i_{4}}\left(x_{0}\right) \ldots R_{i_{2 k-1} i_{2 k}}\left(x_{0}\right) \cdot d x_{0}^{i_{1}} \wedge d x_{0}^{i_{2}} \wedge \cdots \wedge d x_{0}^{i_{2 k-1}} \wedge d x_{0}^{i_{2 k}} \\
& =\frac{1}{2^{k}} \cdot \operatorname{Tr} R^{k} \text {. }
\end{aligned}
$$

This completes the proof of Theorem 10 .

REMARK 3. We recall that, within the theory of linear connections, the closedness of the form $\operatorname{Tr} R^{k}$ in the de Rham complex is a consequence of the Bianchi identity. It is interesting to mention that the same result follows trivially in the context of direct connections as a consequence of three facts: (i) the expression of the curvature of a direct connection depends polynomially only on the functions $\tau(x, y)$ differentiated once or two times, (ii) to each differentiation $\frac{\partial}{\partial x^{i}}$ there corresponds an exterior derivative factor $d x^{i}$, and (iii) the symmetry of mixed partial derivatives (Schwarz lemma).

\section{References}

[C.1] A. Connes, Non-commutative differential geometry, Publ. Math. IHES 62 (1985), 257360.

[C.2] A. Connes, Non-Commutative Geometry, Academic Press, 1994.

[C.M] A. Connes and H. Moscovici, Cyclic cohomology, the Novikov conjecture and hyperbolic groups, Topology 29 (1990), 345-388.

[dR] G. de Rham, Variétés Différentiables, Hermann, Paris, 1960. 
[L] J.-L. Loday, Cyclic Homology, Springer Verlag, 1992.

[M] J. Milnor, Lectures on Characteristic Classes, Princeton Mathematical Studies 76, 1974.

[M.T] A. S. Mishchenko and N. Teleman, Almost flat bundles and almost flat structures, to appear.

[Tc] C. Teleman, Sur le caractère de Chern d'un fibré complexe différentiable, Rev. Roumaine Math. Pures Appl. 12 (1967), 725-731.

[Tn.1] N. Teleman, A geometrical definition of some André Weil's forms which can be associated with an infinitesimal connection, St. Cerc. Math. 18 (1966), 753-762.

[Tn.2] N. Teleman, Distance function, linear quasi connections and Chern character, IHES Prepublications M/04/27, June 2004.

[Tn.3] N. Teleman, Direct connections and Chern character, in: Proceedings of the International Conference in Honor of Jean-Paul Brasselet, Luminy, May 2005.

[Tn.4] N. Teleman, Microlocalisation de l'homologie de Hochschild, C. R. Acad. Sci. Paris 326 (1998), 1261-1264. 